\title{
PROYECTO CURRICULAR DE LICENCIATURA EN ELECTRÓNICA UNA VISIÓN SINTÉTICA
}

\author{
Iván Darío Urrea Ospina \\ Profesor Departamento de Tecnología \\ Coordinador Proyecto Curricular de Licenciatura en \\ Electrónica
}

\begin{abstract}
The present article has as main intention to present a summary of the structure of the Curricular Project of Licentiate in Electronics that was presented to the National Council of Accreditation for previous accreditation, with the purpose of carrying out its popularization and presentation especially to the national academic community. Next they are presented the context in the one which you development the proposal, the relating epistemológico, pedagogic and didactic, the curricular proposal and the investigation component from which the process of formation of Graduates is approached in Electronic.

In the final part a description of the project is presented as for its institutional characteristics, objectives and the competitions that he/she hopes fo achieve in the students that participate in its development.
\end{abstract}

\section{RESUMEN}

El presente artículo tiene como intención principal presentar una síntesis de la estructura del Proyecto Curricular de Licenciatura en Electrónica que fue presentada al Consejo Nacional de Acreditación para acreditación previa, con el fin de realizar su divulgación y presentación especialmente a la comunidad académica nacional.

A continuación se presentan el contexto en el cual se desarrollo la propuesta los referentes epistemológicos, pedagógicos y didácticos, la propuesta curricular y el componente de investigación desde los cuales se aborda el proceso de formación de Licenciados en Electrónica.

En la parte final se presenta una descripción del proyecto en cuanto a sus características institucionales, objetivos y las competencias que espera lograr en los estudiantes que participan en su desarrollo.

\section{INTRODUCCIÓN}

Frente al contexto Nacional y a las comunidades de pedagogos, el Proyecto Curricular de Licenciatura en Electrónica (PCLE) se concibe acorde con las innovaciones que implican la actual política de internacionalización, la búsqueda de una mejor calidad de educación y, en el marco de las sugerencias de la comisión de Ciencia y Tecnología reunida en el año 1993, buscar la construcción de una comunidad de investigadores que según esa proyección debe ser de 36000 para el año $2000^{1}$. Este enfoque, conlleva por lo

\footnotetext{
${ }^{1}$ MEN. Misión Ciencia Educación y Desarrollo. Colombia al filo de la oportunidad. Informe Conjunto. Santa Fe de Bogotá 1994. Pág. 124
} 
tanto a revisar las relaciones que la Universidad Pedagógica Nacional sostiene con el entorno Nacional con miras a que sea la institución llamada a marcar los derroteros a seguir en términos de educación tecnológica.

Desde la historia interna de la Universidad, es importante anotar que las reformas y cambios propuestos durante los últimos años han propiciado condiciones especiales para actualizar el Proyecto Curricular de Licenciatura, de tal manera que se haga más competitivo en cuanto a la misión que le corresponde en la formación de docentes, acorde con las nuevas conceptualizaciones que sobre la Ciencia, la Tecnología y la Pedagogía manejan las comunidades de especialistas en este campo particular de la cultura.

Para dar respuesta a estas expectativas El Proyecto Curricular de Electrónica concibe sus fundamentos conceptuales en los Sistemas Digitales, de Control, de Comunicaciones y de Automatismos, que se estructuran en el diseño y aplicación de materiales que sometidos a campos electromagnéticos permiten desarrollar dispositivos análogos y digitales para el control de sistemas de generación transmisión de energía y masa, y manejos de información.

Desde esta perspectiva, el PCLE se constituye en un aporte al desarrollo social y cultural que atraviesa la nación colombiana, y en un pilar de gran importancia desde el punto de vista económico, partiendo de la proyección de formar ciudadanos comprometidos con el desarrollo tecnológico del país.

En igual manera, esta propuesta surge de las inquietudes de actualización e innovación de un equipo de profesores que trabajaron durante el lapso comprendido entre el año 1995 y 1997 cuando fue aprobado y reconocido institucionalmente; y en el que participaron para su concreción los profesores Carlos Julio Romero Castro, Iván Darío Urrea Ospina, Jorge Darío Rivadeneira, José Manuel Mora Godoy, Rafael Gómez Ballesteros del Departamento de Tecnología de la Universidad Pedagógica Nacional. Sin embargo en su realización se hicieron presentes las inquietudes y aportes de los profesores y estudiantes.

\section{CONTEXTO DE LA PROPUESTA DEL PROYECTO CURRICULAR DE LICENCIATURA EN ELECTRÓNICA}

Asumiendo la concepción de academia implementada en el Proyecto Educativo Institucional $^{2}$, el PCLE se fundamenta, de igual manera, en las funciones básicas de investigación, formación y proyección social; asumiendo como de mayor importancia la función de formación de Licenciados en Electrónica, sin desconocer que tal proceso ha de ser derivado de unas acciones de investigación y que en sí mismo tiene un carácter de proyección social.

Por lo tanto, el PCLE se debe entender como una respuesta a las exigencias de ordenamiento en el tiempo y en el espacio del proceso de formación de Licenciados en Electrónica, mediante la implementación de las políticas, principios, misión, valores, y visión formuladas en el Plan de Desarrollo Institucional cuya proyección, adquiere significado importante en la formación de docentes para el área de Tecnología e Informática en los niveles de Educación Básica, Media Técnica, Tecnológica y Superior,

\footnotetext{
${ }^{2}$ Universidad Pedagógica Nacional. Proyecto Político Pedagógico de la U.PN., Identidad y compromiso Institucional. U.P.N. Santa Fe de Bogotá. 1997.
} 
especialmente desde la consideración de los sistemas electrónicos ya que es la Universidad Pedagógica Nacional, la Institución convocada por la sociedad colombiana para formar los docentes en este sector de la cultura.

Valga la pena mencionar que en cuanto al proceso de formación existe una divergencia bastante amplia en el momento de considerar la formación de un profesional para la Industria, y un docente; aunque existe diversidad de facultades de formación de Ingenieros electrónicos, es el PCLE de la Universidad Pedagógica Nacional uno de los pocos centros académicos especializados en la formación de investigadores en los procesos de aprendizaje y enseñanza del saber electrónico.

En este contexto, el PCLE encamina todos sus mejores esfuerzos para que sea un espacio en el cual se adelanten proyectos de investigación en los procesos de enseñanza y aprendizaje de los diferentes tópicos relacionados con el saber electrónico, para ser soporte fundamental a las instituciones de educación básica y media profesional en lo referente a la implementación de nuevas alternativas de enseñanza de la electrónica, para proponer y desarrollar proyectos de investigación y formación en las áreas educativa y tecnológica que contribuyan al desarrollo de las diversas regiones del país, para posibilitar el Desarrollo de líneas de investigación en electrónica y afines, tanto en lo tecnológico como en lo pedagógico y lo didáctico.

\section{REFERENTES EPISTEMOLÓGICOS}

En el Proyecto Curricular se reconoce la tecnología como un sector autónomo, y en estrecha relación con todos los otros sectores de la cultura. Como discurso, aborda la problemática relacionada con la construcción de conocimiento en torno a los instrumentos, que el hombre elabora para la adaptación, transformación, control, y dominio del entorno que describe como su hábitat, y las implicaciones que ello impone en relación con los individuos y sus relaciones económicas y políticas, con el ambiente y las transformaciones de la ecología como consecuencia de la utilización de nuevos materiales.

Desde esta perspectiva, las ideas centrales sobre las que se realiza la construcción conceptual del proyecto se consideran en los aspectos, derivados de los estudios adelantados por los investigadores y pedagogos del Departamento de Tecnología y de la Facultad de Ciencia y Tecnología de la Universidad Pedagógica Nacional durante los últimos veinte años ${ }^{3}$, entre los cuales es imprescindible destacar los aportes realizados por la Maestría en Pedagogía de la Tecnología, a Maestría: Tecnologías de la información aplicadas a la educación, los adelantos logrados a través de los diferentes espacios de estudio y practicas de los proyectos curriculares de pregrado. Una breve descripción de estas tendencias se describe a continuación.

\footnotetext{
${ }^{3}$ Andrade Londoño, Édgar. El Papel de la Educación en el Desarrollo Nacional de los Países del Tercer Mundo. Santa Fe de Bogotá, Universidad Pedagógica Nacional. Centro de Investigaciones (CIUP).

Gallego Badillo, Rómulo. Et al., 1986. Diseño y evaluación de estrategias y metodologías para la formación científica y tecnológica. Bogotá:

Universidad Pedagógica Nacional, Centro de Investigaciones (CIUP).

Pérez Calderón, Unas. 1989. Educación, tecnología y desarrollo. (Puntos de discusión). Bogotá: Panamericana. 1989. Estudio proyectivo del trabajo de investigación en el departamento de Tecnología. (Informe de Investigación). Bogotá: Universidad Pedagógica Nacional. Centro de Investigaciones (CIUP). Romero O., Carlos Julio y Ortiz Ch., Evelio Nicanor. Area de Tecnología e Informática, U. P N. Santa Fe de Bogotá. 1999. 86
} 
Un enfoque es el de asumir el estudio de la tecnología desde las etapas de desarrollo de este saber. Según esta perspectiva, la tecnología se puede consideran desde los criterios de los materiales usados, los procesos de transformación con los cuales se hace uso de los materiales, y las herramientas empleadas para tales fines. Se consideran igualmente, en este aspecto, las relaciones de intercambios comerciales y las consecuencias que implica la utilización y desarrollo de las tecnologías en sus diferentes acepciones en relación con la distribución del poder político.

En el caso particular del saber electrónico, se consideran la teoría de los semiconductores, y sus procesos de transformación en dispositivos discretos e integrados, así como los conceptos electrónicos implicados en el diseño y construcción de circuitos y sistemas empleados en las diferentes aplicaciones de la vida moderna.

Derivado de estos desarrollos yen consonancia con las políticas actuales de globalización e intercambios económicos y políticos es muy importante describir la evolución y cambios de paradigmas desarrollados en el saber electrónico, así como sus implicaciones en la cultura, la sociedad y las teorías y métodos elaborados para enseñarlos y aprenderlos.

Otra alternativa es la que hace referencia a la estructura del saber tecnológico desde los campos discursivos que implican sus diferentes especialidades y sus necesarias relaciones intra, inter y multidisciplinares Teniendo en cuenta los anteriores referentes, la tecnología, se puede entender, de una parte, como el campo que explica y desde el cual se pueden diseñar los sistemas automatizados; desde otra, el problema de los materiales.

Los Sistemas Automatizados hacen referencia a los sistemas que involucran las relaciones entre los mecanismos, los censores y los controladores. Estos sistemas se pueden contemplar, para su análisis, desde dos campos: los sistemas mecánicos desde el cual se modelan y diseñan los problemas tecnológicos relacionados con la generación y conversión de energía; transmisión de potencia, sistemas de mecanismos; y control de potencia mediante la utilización de sistemas de fluidos, hidráulicos y neumáticos. Los sistemas de control especifican los campos discursivos con los cuales se modela y diseña el control desde la óptica de los automatismos; en este ambiente se enfatiza especialmente en los sistemas electrónicos, los sistemas hidráulicos y los sistemas neumáticos de control; aunque en algunos casos se emplean sistemas de mecanismos como elementos de control. En lo que hace referencia a los sistemas electrónicos se explicitaría que son los sistemas con los cuales se ejerce control sobre los sistemas mecánicos bajo el principio de automatización, y de igual manera en la referencia a los sistemas de control, de manejo de datos para realizar control a distancia (telecontrol) o transmitir información a grandes distancias en el campo de las comunicaciones y el campo de producción y manejo de información (Software) con sistemas electrónicos como soporte (Hardware); la combinación de estos dos elementos permitirá explicar los procesos o sistemas de computación análogos y digitales.

El sistema de los materiales hace referencia a la construcción de nuevas estructuras con las cuales diseñar nuevos instrumentos. En ella están implicados los procesos en los cuales intervienen el trabajo con estructuras orgánicas, la Biónica, la Biomédica, y La Tecnología Genética. Las diferentes explicaciones de transformación y elaboración de materiales: la Metalurgia, la lónica, y la Polimerización.

Entre los dos elementos, los de los automatismos y los materiales, las relaciones son también de doble sentido en la medida que para diseñar sistemas automatizados es 
necesario contar con materiales específicos, y para los procesos de producción de materiales es indispensable contar con los sistemas automatizados. En otras palabras, cada uno de los Proyectos de investigación está estrechamente vinculados tanto en su base conceptual como en su dependencia operativa.

Desde esta perspectiva en el Proyecto Curricular, la tecnología electrónica se entiende como el entramado conceptual de tres elementos: Los Sistemas de Comunicaciones, Los Sistemas de Control, y La Electrónica de Potencia; cimentados sobre los, principios fundamentales del diseño digital y análogo, los fundamentos de automatización, y la concepción del diseño tecnológico.

Finalmente, desde la alternativa filosófica y epistemológica se puede afirmar que durante los últimos tiempos, se han desarrollado bastantes desarrollos conceptuales especialmente en cuanto a la tecnología se hace referencia. En cuanto a la concepción que se asume en el Proyecto Curricular de Licenciatura en Electrónica es la desarrollada pon un equipo de profesores de la Facultad de Ciencia y Tecnología de la Universidad Pedagógica Nacional ${ }^{4}$ durante los años ochenta y que se ha enriquecido durante el transcurso y evolución de los trabajos de investigación, las innovaciones y actualizaciones de los proyectos curriculares y las practicas pedagógicas y didácticas.

Desde esta óptica, se asume que el saber tecnológico se halla constituido por los elementos: Sistema Tecnológico, Modelo Tecnológico, Diseño Tecnológico, Prototipo, Reglas de Producción y Las relaciones de doble sentido que entre ellos se establecen. Desde esta estructura se aborda y explica la organización de los diferentes elementos que implican construir una teoría tecnológica.

El saben electrónico, en el Proyecto Curricular de Licenciatura en Electrónica se aborda desde los Sistemas de Comunicaciones, y los Sistemas de Control cada uno de ellos entendido como desarrollo de la combinación de modelos análogos y digitales que permiten la elaboración de sistemas inteligentes ya sea para controlar sistemas de potencia o para permitir el control a distancia de sistemas automáticos.

En este sentido, una descripción de la estructura de estas líneas de estudio debe comprometer los conceptos relacionados con la fundamentación matemática y física que implican la realización de los modelos electrónicos que describen los intercambios de energía entre los diferentes dispositivos que componen los circuitos, así como la formulación de procesos de transformación de los materiales para elaborar los diferentes dispositivos.

En una siguiente etapa de conformación de la estructura conceptual, se consideran los conceptos tecnológicos implicados en la descripción de las dos líneas de trabajo más relevantes: los modelos análogos y digitales, en los cuales se implican el conocimiento sobre la comprensión del funcionamiento y la estructura de dispositivos derivados de las tecnologías de integración física de componentes discretos en pastillas de silicio y germanio, a partir de los cuales se hace posible comprender la estructura de la arquitectura de sistemas complejos utilizados como soporte para procesos de comunicaciones, control y procesamiento de información.

En cuanto al Diseño Tecnológico, parte central del Proyecto Curricular, se le considera como el elemento epistemológico con el cual se da cuenta de la transformación de los

\footnotetext{
${ }^{4}$ Ver referencias citadas previamente. 
modelos lógicos matemáticos en tecnofactos; es decir, la materialización de las ideas como resultado de la aplicación del operador diseño tecnológico sobre las estructuras propuestas en los modelos tecnológicos.

En la particularidad del saber electrónico se considera el diseño como el espacio de reflexión que permite realizar la transición desde modelos lógico-matemáticos hacia circuitos concretos que poseen las propiedades de ser elaborados con materiales específicos, poseer una estructura interna y la propiedad de realizar transformaciones de energía, cada una de ellas en intensidad y en proporción con la función específica en que han de ser usados.

Es por tanto necesario postular que existen dos paradigmas fundamentales de diseño electrónico; el diseño análogo y el diseño digital. El primero parte de los modelos derivados del tratamiento de las señales electromagnéticas continuas en el tiempo según los algoritmos de las ecuaciones diferenciales y los teoremas derivados para el caso particular del saber electrónico; el segundo parte de los postulados de la lógica formal y las álgebras binarias para representar el comportamiento de señales discretas para posibilitar el análisis y la síntesis de operadores lógicos para el procesamiento de información y que se concretan usualmente en pastillas de circuitos integrados.

Como puente de enlace entre los dos paradigmas mencionados, ha surgido un campo de prominente investigación y desarrollo conceptual y tecnológico, en el cual se tiene como objeto de estudio los sistemas conversares de análogo a digital y viceversa, dispositivos transductores de energía, sensores y actuadores, y dispositivos de visualización entre otros.

\section{REFERENTE PEDAGÓGICO Y DIDÁCTICO}

El Proyecto Curricular tiene como eje central de acción la investigación sobre el proceso de formación de los nuevos Licenciadas en Electrónica, desde las conceptos fundamentales hasta los más especializados que el Proyecto Curricular prepare como metas de logra, y que de alguna manera son convalidadas par la comunidad de especialistas en este campo del saber. Igualmente, es una atenta de proyecto de vida pedagógica, didáctica, social, epistemológica y axiológica para el estudiante, en concordancia con las exigencias de la actualidad nacional. Igualmente, pretende ofrecen un camina mediante el cual las estudiantes que se encuentran adscritos a él, tengan un acceso a la comunidad de profesionales de la electrónica.

El problema de la construcción de nuevas conocimientos en el interior de la comunidad de electrónicas lo realizan las miembros de mayor experiencia, quienes en el desarrollo de sus actividades contribuyen en la formación de los miembros noveles en las distintas actividades, tanto conceptuales como prácticas, durante el desarrollo misma de sus actividades especializadas; sin embargo, para que esto sea posible, es indispensable que los nuevas miembros reconozcan los fundamentas, lenguajes, y prácticas básicas que durante el intercambio se hacen necesarias. Es en este punto donde el desarrollo del presente Proyecto Curricular adquiere importancia vital para la inscripción de los aspirantes a ser miembros de la comunidad. Dicha inscripción epistemológica en el saber electrónica se lleva acabo mediante la realización de un trabajo pedagógico adelantado con los estudiantes, y mediante el cual se propicie la autoconstrucción de los saberes electrónicos. 
En este orden de ideas, el principio de acción de importancia de primer orden del Proyecto Curricular no es la creación de nuevos conocimientos del saber específico, sino reconocer que este es precisamente el media adecuada a través del cual se adelante la tarea de formación profesional de las personas que aspiran a ser miembros de la comunidad, mediante la implementación de diferentes proyectos de investigación formativa.

El intercambio conceptual que se asume en el Proyecto Curricular de Licenciatura en Electrónica, parte de la formulación y reconocimiento del hecha de que el saber especifico desarrollado en el interior de la comunidad de especialistas es una concepción de universa en la cual se implican todos los aspectos can que se pudiera definir un ser humana y desde otra punto de vista que en los diferentes grupos humanos existen personas interesadas en pertenecer a dichas colectividades como miembros activos. De esta polaridad surge la necesidad de elaborar un discurso que de cuenta de los procesos de enlaces entre los especialistas productores de conocimiento y los noveles interesados en acceder a él, la pedagogía y la didáctica.

Este planteamiento, de alguna manera, presenta das formas de acatar el problema: una es la denominada bajo el concepto de aprendibilidad, en la cual se asume que el estudiante ingresa intencionalmente al proceso con la idea fundamental de reconstruir, a construir las relaciones e interacciones conceptuales que le permitan discurrir acerca del universa como lo hacen los que formulan las teorías y postulados tecnológicos; de igual manera ellos arriban con la idea de interactuar en el mundo comprometidos con las circunstancias del entorno en el cual habitan y sobre el que deben proyectar acciones asumidas como válidas.

Otra forma de abordar el problema tiene que ver can las concepciones de quienes se dedican al problema de la enseñanza de la tecnología en particular y en general de los saberes; se encuentra que es importante conocen con alto grado de profundidad las producciones que circulan como válidas en las comunidades de especialistas, así como aquellos aspectos en las cuales existen querellas académicas y a los cuales hay que dedicar esfuerzos y trabajo para intentar darles solución. De otra manera, el docente debe ser poseedor de un acervo que le permita mediar con sus estudiantes en cuanto al intercambio conceptual, metodológico, actitudinal y axiológico, lo cual implica que el postulada de que un saber es enseñable rebasa, incluso las detalles relacionados con la producción del saber y se constituye en centro de atención y dedicación especial de investigaciones acerca de cómo aprenden los seres humanas.

En este orden de ideas, el proceso de formación de nuevos estudiosos y practicantes del saber electrónico se debe llevan a cabo mediante el análisis y síntesis de problemas básicos desarrolladas en complejidad ascendente, siguiendo las metodologías de Solución de Problemas o de elaboración de Proyectos. Es en este punto donde el concepto de diseño electrónico se hace importante como eje central del Proyecto Curricular, pues el trabajo pedagógico se ha de llevan a cabo en la reconstrucción de los problemas básicos mediante los cuales se haga posible la inscripción por parte del estudiante en el uso del lenguaje especializado, el desarrollo de las actividades técnicas indispensables para elaborar las soluciones y la lectura y producción de artículos especializados.

En el desarrollo del procese de formación se articulan todos los elementos del Proyecto Curricular incluyendo los de las Areas De Pedagogía Y Didáctica, de Investigación y 
Deontológicas, en la medida que los desarrollos de los problemas deben ser enfocados a conceptualizar las soluciones con una visión universal.

Finalmente, el asumir esta metodología implica reconocer que el campe del saber electrónico, en las especifidades que como campos de investigación se proponen, es viable de enmarcar dentro de la estructura epistemológica mencionada anteriormente; en términos más específicos implica tener en cuenta los siguientes aspectos:

1. La elaboración de los distintos circuitos que una aplicación particular requiere, obedece-a las variables de modelos matemáticos, incluso si se deja de lado por ahora la problemática que plantea la construcción de pastillas de circuitos integrados. En el casa más elemental, los circuitos siguen las lógicas del álgebra de Boole o binarios, hasta los casos en los cuales se hace indispensable trabajar con los complicados modelos de sistemas retroalimentados que siguen las geometrías de los campos vectoriales y las ecuaciones diferenciales, para dar configuración a los cuerpos de ecuaciones que luego darán cuenta de los sistemas de control automático en unos casos, y en otros a las transmisiones y recepciones de energía en forma de campos electromagnéticos.

2. El proceso de diseño de circuitos conlleva a dar cuenta de las relaciones de forma, función y estructura del diseño tecnológico aplicado a este campo particular, así como de la minimización de los consumos energéticos sin sacrificar eficiencia. En otras palabras, es especificar las relaciones que cada uno de los elementos sostiene con el espacio que han de ocupar sobre una placa y parlo tanta el empleo y racionalización de materiales.

3. En cada uno de los circuitos que se deseen diseñar o rediseñar, están implicados los materiales, especialmente observados desde su comportamiento cuando son sometidos a campos electromagnéticos y que por la tanto es indispensable ser conocedor de tales estructuras, desde los planteamientos que la física del estado sólida sugiere como modelas; materiales, que el tecnólogo ha de transformar para que en nuevas disposiciones se comporten de acuerdo a los requerimientos que el diseño impone.

4. Cada uno de los prototipos de control guarda una relación de correspondencia con las sistemas de potencia que se desean controlar y que por lo tanta es necesario la constitución de grupos interdisciplinarios de trabajo, en los cuales la especialidad de cada uno de sus miembros adquiere vital importancia para el logro de los tecnofactos.

5. En la elaboración de un tecnofacto, como resultada de un Proyecto Curricular o un proyecto de investigación, están necesariamente involucrados los procesos de producción; parlo tanto, es necesario conocerlos asumirlos y practicarlos, desde las perspectivas de la construcción en sí y desde la necesidad de realizar trabajo experimental para determinan las posibles valores que las variables del modelo han de tomar en la concreción del tecnofacto, como en la necesidad de elaborar cartas técnicas y dirigir y/o asesorar los trabajos técnicos especializados que implica tal elaboración.

6. Estos planteamientos sugieren la necesidad de unas normas de producción en el interior de los grupos de investigación tecnológica, las cuales se sostienen principalmente en el hecho de que la lectura y escritura que los miembros del grupo realicen sobre una estructura en particular tengan significados afines. 


\section{PROPUESTA CURRICULAR}

La formulación del PCLE se inscribe dentro del espíritu planteado en el Proyecto Educativo Institucional, aunque conservando las relaciones y distancias pertinentes a la especifico de su propuesta. En esta misma idea, a través de la formulación del referente conceptual y de los componentes del plan de estudios, el PCLE recoge de manera particular lo expresado en el Proyecto Educativo Institucional en cuanto a los aspectos metodológicas de organización académica.

En relación con los ambientes educativos, y retomando los planteamientos del Proyecto Educativo Institucional, como aquellas "expresiones culturales o los saberes que, integradas, conforman la cultura que va a caracterizar la identidad, así como al compromiso, la pertenencia y la imagen del futuro maestro", el PCLE se desarrolla en los siguientes ambientes de formación.

Ambiente de formación disciplinar específica, en el PCLE, se estudia y contrastan experimentalmente las aspectos relacionados con los sistemas electrónicos análogos y digitales; profundizadas a partir de la concepción del diseño electrónico y desde la cual se reconstruyen los modelos de la tecnología del estado sólido aplicado a elementos de circuitos y estructuras de mayor funcionalidad desde el punto de vista de la transformación de la energía.

Corresponde a la formación científica, tecnológica y técnica, del PCLE; tiene como ejes principales de atención conceptual los sistemas electrónicos análogos y los sistemas electrónicos digitales. De las necesarias relaciones conceptuales entre estos dos sistemas surgen múltiples maneras de especialización del conocimiento electrónico, de las cuales el PCLE aborda los Sistemas de Comunicaciones, los Sistemas de Potencia y los Sistemas de Control.

Ambiente de formación científica e investigativa, en este ambiente se involucra durante los dos ciclos de implementación del PCLE, en el ciclo de fundamentación el estudiante accede a los fundamentos del saben electrónico y a sus pedagogías y didácticas a través de diferentes actividades formativas, para luego integrarlos en los diferentes procesos de reconstrucción y construcción de estos saberes en el ciclo de profundización, en el cual las estudiantes inician como primera práctica investigativa, un trabajo de elaboración particular inscrito en las líneas de investigación del PCLE.

El ambiente de formación pedagógica se constituye en una de los ejes centrales del PCLE, evidenciada en el desarrollo de los seminarios de pedagogía, en las cuales se elabora y reconstruye el discurso de la pedagogía y didáctica del saber electrónico en el ciclo de profundización, para luego ser llevados a la realidad concreta del aula en la práctica pedagógica y didáctica y en el trabajo de grado.

De igual manera, inscribe las componentes de la práxis y reflexión pedagógica y didáctica en el campo específico de la tecnología electrónica y sus componentes técnicas en la mira de lograr una proyección educativa exitosa; de esta manera, el área de Pedagogía y didáctica se constituye en otro eje Curricular importante el PCLE.

El ambiente de formación deontológica y en valores humanos se estructura a lo largo del plan de estudios del PCLE y como una parte central de atención desde todos y cada uno de sus componentes, se hace énfasis en las propuestas de formación de valores, identidad y compromiso como han sido enunciadas en la formulación filosófica del PCLE. 
Abarca los aspectos de reflexión acerca del entorno social y las relaciones que existen entre él y la aplicación de conocimientos específicos de su interior. De manera específica, el área de integración da cuenta de la formación de valores, del compromiso ético y de las relaciones que existen entre el saber especifico con el entorno social, a partir de los cuales el estudiante construye su identidad y se proyecta en el entorno de la sociedad como un agente potencialmente en capacidad de realizar transformaciones y proponer soluciones.

En igual forma, corresponde a los aspectos que tienen que ven con los procesos de formación estética y deportiva de los estudiantes.

Estos ambientes de formación se desarrollan en dos ciclos, un Ciclo de Fundamentación, y un Ciclo de Profundización. Distribuidos en cuatro y seis semestres de actividad académica respectivamente.

En ciclo de fundamentación se establecen las relaciones de apropiación de los fundamentos conceptuales desde las ciencias y la tecnología, a partir de los estudios correspondientes a las fundamentos de matemáticas y de física indispensables para acceder a la construcción del discurso electrónico; también se realiza el primer acercamiento a la epistemología de la tecnología y sus bases técnicas en el espacio de fundamentas de tecnología, así como el desarrollo de las bases conceptuales del discurse electrónico conocidos como circuitos; en este espacio es donde se realiza la primera integración entre la matemática, la física y el análisis del comportamiento de los materiales sometidos a campos electromagnéticos. En cuanto a la formación pedagógica se trabajan los seminarios que relacionan la educación, la sociedad, la economía, la política y la cultura relaciones con la sociedad, la economía y la cultura.

En el ciclo de profundización se considera en das momentos de formación; el primera de ellos se ha denominado etapa de profundización, en la cual se desarrollan los aspectos relacionados con el diseño electrónico, se adelanta en cuatro semestres, y el segunda de ellas se ha llamado etapa de Síntesis, en la cual el estudiante elabora una propuesta pedagógica y didáctica, a través de las actividades de la práctica pedagógica y didáctica y el trabajo de grado, desarrollada durante los das últimas semestres del proyecto.

De manera específica, la etapa de profundización es la etapa en la cual se estudian y contrastan experimentalmente los aspectos relacionados con los sistemas electrónicos digitales, y análogos; así mismo se estudia lo relacionado con la fundamentación en investigación en pedagogía y didáctica. Corresponde a los semestres quinto a octavo del desarrollo del plan curricular.

En esta etapa se ha de profundizar en el discurso del Diseño Electrónico, estudiado desde las concepciones de Diseño análogo y Diseño Digital. El elemento de esta estructura, que establece la relación entre esta etapa y la anterior es el denominado Circuitos; en él se realizaran las precisiones pertinentes desde los modelos Físico matemáticos hacia la Electrónica, así como las pertinentes entre la Electrónica y el Diseño, especialmente en los estudios de análisis y síntesis de circuitos.

Bajo la concepción de Diseño Electrónico, en esta etapa se realizan los modelos de la tecnología del estado sólido aplicada a elementos de circuitos, y los fundamentas iniciales de diseño de estructuras funcionales desde el punto de vista de transformación de energía eléctrica, (amplificadores y osciladores). 
En el ambiente de Pedagogía y didáctica se desarrollan los seminarios en las cuales se construye el discurso de la pedagogía y la didáctica; y el ambiente de Formación deontológica y en valores humanos establecen las relaciones entre la tecnología y la ciencia, la sociedad, la política y sus implicaciones en Colombia.

Las necesarias relaciones que se deben establecer entre los ambientes de formación están mediadas por la discusión entorno a la tecnología, y específicamente en lo relacionado con Sistemas Electrónicos. Si bien es cierto que lo específico ha de discurrir entorno a problemas puntuales sobre el saber electrónico, lo pedagógica y didáctica ha de discurrir acerca de la construcción epistémica de tales conceptos así como de sus relaciones con el entorno social para el cual se proyectan.

La etapa de síntesis es la etapa en la cual se aborda la proyección individual e interdisciplinania, para orientar los procesos de especialización en campos específicos del saber electrónico; así misma lo relacionada con la realización de propuestas pedagógicas y didácticas, a través de la realización del trabajo de grado. Corresponde a los tres últimos semestres del plan curricular.

El ambiente de formación especifica, en este momento del PCLE concluye con una síntesis de los modelos desarrollados en los ciclos anteriores estudiando de manera especial los sistemas de control y de comunicaciones. El ambiente de formación pedagógica concluye con la puesta en acción de los saberes elaborados en durante el desarrollo del proyecto, mediante el diseño e implementación de estrategias para la enseñanza de los conceptos electrónicos, así como de propuestas de solución a problemas que se encuentran en el aula de clase, los cuales se han de proponer en términos de trabajos de investigación y serán plasmados en un trabajo de grado.

\section{LA INVESTIGACIÓN}

En este aspecto es importante resaltar que durante los últimas años, en el PCLE, se han adelantado diferentes trabajos de grado orientados por intereses e intenciones particulares de los profesores y estudiantes, y que sin embargo hasta el momento no ha sida posible formular de manera explícita y estricta, líneas y proyectos de investigación; sin embargo con las concepciones planteadas a lo largo del presente documento y retomando como punto de partida los tópicos enunciados en los trabajos citados se enuncian como punto de partida tres campos de estudio en los cuales formalizar la línea de investigación del PCLE.

El primero, el campo estudio en pedagogía y didáctica del PCLE, contra su atención en la formulación de trabajos puntuales sobre la elaboración de estrategias y metodologías para la enseñanza y el aprendizaje de los conceptos electrónicas; sobre estudios en desarrollo curricular y en ambientes de aprendizaje, dirigidos de manera especial a dar solución a las necesidades de formación de estudiantes de la Educación Básica, Media Técnica y Formación Superior.

El segundo, es el campo estudio en modelos y prototipos se orienta al diseño y elaboración de tecnofactos electrónicos mediante los cuales proponer soluciones a diversas necesidades de la industria en la producción de bienes y servicios, tanto en tarjetas de circuitos como en programas de automatización y control. Este campo se inscribe en los desarrollos que se propongan desde los estudios realizados en el área específica de formación. 
Y el tercer campo de estudio en material didáctico, se orienta a diseñar y elaborar prototipos de material de apoyo, en equipos o en programas informáticas, necesarios para la enseñanza de los diferentes conceptos electrónicos, especialmente siguiendo los requerimientos de las necesidades impuestas en la Educación Básica, Media Técnica, Tecnológica y Superior.

\section{DESCRIPCIÓN DEL PROYECTO}

Titulo:

Registro ICFES:

Norma interna que lo crea:

Departamento:

Facultad:

Lugar de ejecución:

Ciudad:

Titulo que otorga:

Escolaridad:

Dedicación:

Duración:
Proyecto Curricular de Licenciatura en Electrónica 110545373701100111100

Acta \#14 del Conseja Académico (agosto de 1997)

Tecnología

Ciencia y Tecnología

Universidad Pedagógica Nacional

Santa Fe de Bogota

Licenciado en Electrónica

Presencial

Dedicación de tiempo completo

Diez semestres académicos

\subsection{Objetivos}

Son objetivos del PCLE:

- Formar licenciados en electrónica, can los basamentos indispensables para abordar procesos de enseñanza aprendizaje en el saber de la tecnología y especialmente en lo relacionado con el saber electrónico.

- Fomentar las actividades de investigación en el campo de la pedagogía y didáctica de la tecnología, y de la tecnología electrónica en particular, para la solución de problemas regionales y nacionales.

- Promover el desarrollo y difusión del conocimiento en pedagogía y didáctica de la tecnología electrónica.

- A partir de una integración del saber pedagógico y didáctico con el saber tecnológico en el campo de la electrónica, atender los diferentes niveles educativos del país.

\subsection{Competencias que se espera lograr}

Las competencias que se espera que construyan los estudiantes del PCLE son:

- Su capacidad para integrar en su práxis profesional el desarrollo de estrategias pedagógicas y didácticas, con actividades que permitan solucionar problemas de índole tecnológica.

- Su conocimiento y capacidad de acción frente a situaciones de tipo humanístico especialmente en aquellos casos en las cuales su desempeño profesional este orientado a la formación de niñas y adolescentes; no olvida que la producción tecnológica es realizada por seres humanos y para seres humanos. 
- Manejar los basamentos indispensables para iniciar investigaciones en lo relacionada con pedagogía y didáctica de la tecnología en general y del saber electrónico en particular.

- El desarrollo de su capacidad intelectual y motriz, lo cual le posibilitará articular en su práxis profesional lo concerniente al diseño, construcción, operación y mantenimiento de sistemas electrónicos, en la producción de bienes y servicios.

\section{BIBLIOGRAFÍA}

Andrade Londoño, Édgar. El papel de la educación en el desarrollo nacional de los países del Tercer Mundo. Santa Fe de Bogotá: Universidad Pedagógica Nacional. Centro de Investigaciones (CIUP).

Gallego Badillo, Rómulo. et. al., 1986. Diseño y evaluación de estrategias y metodologías para la formación científica y tecnológica. Bogotá: Universidad Pedagógica Nacional, Centro de Investigaciones (CIUP).

Kuhn, T. S. 1971. La estructura de las revoluciones científicas. México: Fondo de Cultura Económica.

Lakatos, Y. 1981. La metodología de los programas de investigación científica. Madrid: Alianza Editorial.

MEN. Misión Ciencia Educación y Desarrollo. COLOMBIA AL FILO DE LA OPORTUNIDAD. Informe Conjunto. Santa Fe de Bogotá, 1994, pág. 124.

Pérez Calderón, Urías. 1989. Educación, tecnología y desarrollo. (Puntas de Discusión). Bogotá: Panamericana.

- Estudio proyectivo del trabajo de investigación en el Departamento de Tecnología. (Informe de Investigación). Bogotá: Universidad Pedagógica Nacional. Centro de Investigaciones (CIUP).

Romero C., Carias Julia y Ortiz Ch., Evelio Nicanor. Área de Tecnología e Informática. U. P. N. Santa Fe de Bogotá. 1999.

TOULMIN, S., 1977. La comprensión humana. Vol. 1. El uso colectivo y la Evolución de los conceptos. Madrid: Alianza Editorial.

Universidad Pedagógica Nacional. Proyecto Político Pedagógico de la U. P. N., Identidad y compromiso Institucional. U.P.N. Santa Fe de Bogotá. 1997. 